\title{
Outage Performance of Full-Duplex Relay Networks Powered by RF Power Station in Ubiquitous Electric Internet of Things
}

\author{
Yu Zhang $\mathbb{D}^{1}{ }^{1}$ Xiaofei Di $\mathbb{D}^{2}{ }^{2}$ Hongliang Duan $\mathbb{D},,^{3,4}$ Xi Yang $\mathbb{D}^{5,6}$ Peng $W u\left(\mathbb{D},{ }^{1}\right.$ \\ and Baoguo Shan $(10)^{1}$ \\ ${ }^{1}$ Institute of Economics and Energy Supply and Demand, State Grid Energy Research Institute Co., Ltd., Beijing 102209, China \\ ${ }^{2}$ School of Software Engineering, Beijing Jiaotong University, Beijing 100044, China \\ ${ }^{3}$ School of Mechanical Engineering, Northwestern Polytechnical University, Xi'an 710072, China \\ ${ }^{4}$ Beijing Institute of Space Long March Aerospace Vehicle, Beijing 100076, China \\ ${ }^{5}$ School of Information, Beijing Wuzi University, Beijing 101149, China \\ ${ }^{6}$ Beijing Intelligent Logistics System Collaborative Innovation Center, Beijing 101149, China
}

Correspondence should be addressed to Xiaofei Di; xfdi@bjtu.edu.cn

Received 31 March 2021; Revised 17 June 2021; Accepted 16 July 2021; Published 10 August 2021

Academic Editor: Yuan Ding

Copyright (c) $2021 \mathrm{Yu}$ Zhang et al. This is an open access article distributed under the Creative Commons Attribution License, which permits unrestricted use, distribution, and reproduction in any medium, provided the original work is properly cited.

\begin{abstract}
This paper investigates a full-duplex relay network assisted by radio frequency (RF) power station in ubiquitous electric Internet of Things (UEIoT), where a wireless sensor on high voltage transmission line collects electric data and then transmits data to a destination via a full-duplex decode-and-forward relay. The destination receives and processes the data to inspect the state of high voltage transmission line. Both the sensor and relay do not have fixed energy source; so, they first have to harvest energy from a RF power station and then complete information transmission by using the harvested energy. As the line-of-sight (LOS) propagations often exist in outdoor application scenarios of UEIoT, the Rician fading channel is adopted in the network. To explore the system outage performance, the explicit expressions of the outage probability and throughput of the system are derived by using mathematical analysis. Simulation results validate the correctness of our theoretical analysis and also evaluate the effects of system parameters and deployment position of power station on the outage performance.
\end{abstract}

\section{Introduction}

The ubiquitous electric Internet of Things (UEIoT) aims to realize the interconnection of all things and human-computer interaction in the power system by integrating modern information technologies, including wireless sensor networks, artificial intelligence, and advanced communication technologies [1]. The State Grid Corporation of China has planned to initially build UEIoT by 2021 to support the development of grid business and some other emerging businesses. By 2024, UEIoT will be established to form an energy internet ecosystem with joint construction, cogovernance, and sharing $[2,3]$. UEIoT has attracted much attention from academia to industry $[4,5]$.
The UEIoT can be applied to a large number of outdoor and field application scenarios, such as field power line inspection. Unfortunately, although wireless sensors are deployed on the high-voltage line, they cannot be driven directly by the power of the high-voltage line. So, recently passive Internet of Things [6] has attracted attention and become an important part of UEIoT, where the sensors can be wirelessly charged by harvesting energy of the radio frequency (RF) signals emitted by wireless power stations [7-9]. The authors in [7] realized a practical wireless powered sensor network and investigated the efficiency of RF energy harvesting (EH). In [8, 9], an IoT powered by a static and mobile base station was, respectively, studied, where a number of wireless sensors first harvested 
energies from base station and then transmit their information to the base station. The works in [6-9] considered the linear EH model, while $[10,11]$ further considered a more practical nonlinear EH model. In [10], a wireless powered cognitive radio sensor network was studied, where two primary users communicate to each other with the help of a sensor node with nonlinear energy harvester and accurate expressions of the outage probability and throughput were derived for the primary system. [11] considered a downlink nonorthogonal multiple access (NOMA) network, where the near user was equipped with a nonlinear energy harvester, and the outage probabilities and throughput for both the far and near users were derived.

On the other hand, to complete information delivery, wireless sensors generally communicate by using relaying mode due to the long distance among sensors in IoT [12]. Compared with traditional half-duplex relay, full-duplex relay can use the same frequency band to simultaneously receive and transmit information; so, the transmission delay and system spectrum efficiency are greatly improved $[13,14]$. Owing to these merits, full-duplex relay has attracted increasing interest [15-17]. In [15], an optimal beamformer design was proposed for a full-duplex decode-and-forward (DF) relay system with self-energy recycling. [16] extended the one-way relay system in [15] to two-way relay one and analyzed outage performance of the system. The authors in [17] studied effect of residual self-interference (SI) on the stability performance of the full-duplex amplify-and-forward (AF) relay system.

To inherit the benefits of RF EH and full-duplex relaying, some exiting works have investigated the full-duplex relay networks powered by RF EH, see e.g., [18-21]. In [18], some full-duplex AF relays first harvested energy from a source and then received and forwarded the information of the source to a destination by using the harvested energy. The achievable information rate was maximized by designing optimal power allocation and relay selection policy [18]. The authors in [19] further studied two-way relay system and derived closed-form expressions of the throughput and outage probability over Rayleigh fading channels. In [20], a multidestination relay network was considered, where a source first emitted energy to a full-duplex DF relay and then the relay further used the harvested energy to forward the information of the source to multiple destinations, and the spectral efficiency of system was enhanced by jointly optimizing the time allocation, subcarriers, and transmission power of the source and relay. Different from the works [18-21] considered a relay network consisting of a source, a DF relay and a destination, as well as a power station, where the source and relay first harvested energy from the power station and then cooperatively transmit information to the destination.

This paper also considers a full-duplex relay network assisted by a dedicated power station in the UEIoT, as shown in Figure 1. A wireless sensor $(S)$ on high voltage transmission line collects electric data and then transmits data to a base station (D) via a dual antenna full-duplex DF relay $(R)$. $D$ receives and processes the data to inspect the state of high voltage transmission line. Both $S$ and $R$ do not have fixed energy source; so, they first have to harvest energy from a RF power station and then complete information transmission by using the harvested energy. Different from [21], the Rician fading channel model is better suited in outdoor roadside scenario with RF energy harvesting, according to the experimental result [22]. As UEIoT is usually deployed in outdoor roadside scenario, the Rician fading channel model is adopted in this paper.

For such a network, our contributions are summarized as follows. Firstly, the EH process and information transmission are modeled for the full-duplex relay network powered by RF power station. Then, the explicit expressions of the outage probability and throughput of the system are derived by using mathematical analysis in order to explore the system outage performance. Simulation results validate the correctness of our theoretical analysis, and the effects of system parameters on the outage probability are also discussed. Finally, the optimal deployment position of power station is also investigated, which may provide some useful insights on the future practical applications of UEIoT.

The rest of this paper is organized as follows. In section 2, the system model is presented. Section 3 derives the outage probability and throughput of the system. Section 4 shows some simulation results to evaluate our theoretical analysis and illustrate the system performance. Finally, this paper is concluded in section 5 .

\section{System Model}

We consider a full-duplex relay network assisted by RF power station (PS) in UEIoT, as shown in Figure 1, which is composed of a source $(S)$, a relay $(R)$, and a destination $(D)$, as well as a dedicated RF PS. $S$ desires to transmit information to $D$ with the help of $R$. $S$ and $R$ do not have fixed energy source; so, they have to harvest energy from PS and then use the harvested energy to accomplish information transmission. $R$ adopts the decode-and-forward (DF) protocol. As the decoding processing power required at $R$ is much less than its transmit power, its decoding processing power is thus ignored. PS, $S$, and $D$ are equipped with single antenna. $R$ is equipped with two antennas, one is the receiving antenna used to receive information from $S$ or harvest energy from PS, and the other is the transmitting antenna used to forward received information to $D$ in the full-duplex mode.

Figure 2 illustrates the time frame of the transmission protocol. It is assumed that each time frame is with the time period of $T$, which is divided into two parts, where the first part is used for energy transfer and the second one is for information transmission with full-duplex relaying. Considering there exist line-of-sight (LOS) propagations between the nodes in the outdoor UEIoT, the links between any two nodes are modeled as Rician fading channels. The channel coefficients are assumed to be unchanged in each time frame $T$, but may change independently between any two adjacent time frames.

Specifically, during the first part with time interval of $\alpha T$, PS broadcasts energy to $S$ and $R$, where $\alpha$ is the time division factor satisfying $0 \leq \alpha \leq 1$. The energy harvested by $S$ and $R$ can be, respectively, expressed by

$$
E_{S}=\alpha \operatorname{T\eta } P_{B} d_{1}^{-m}\left|h_{1}\right|^{2}
$$




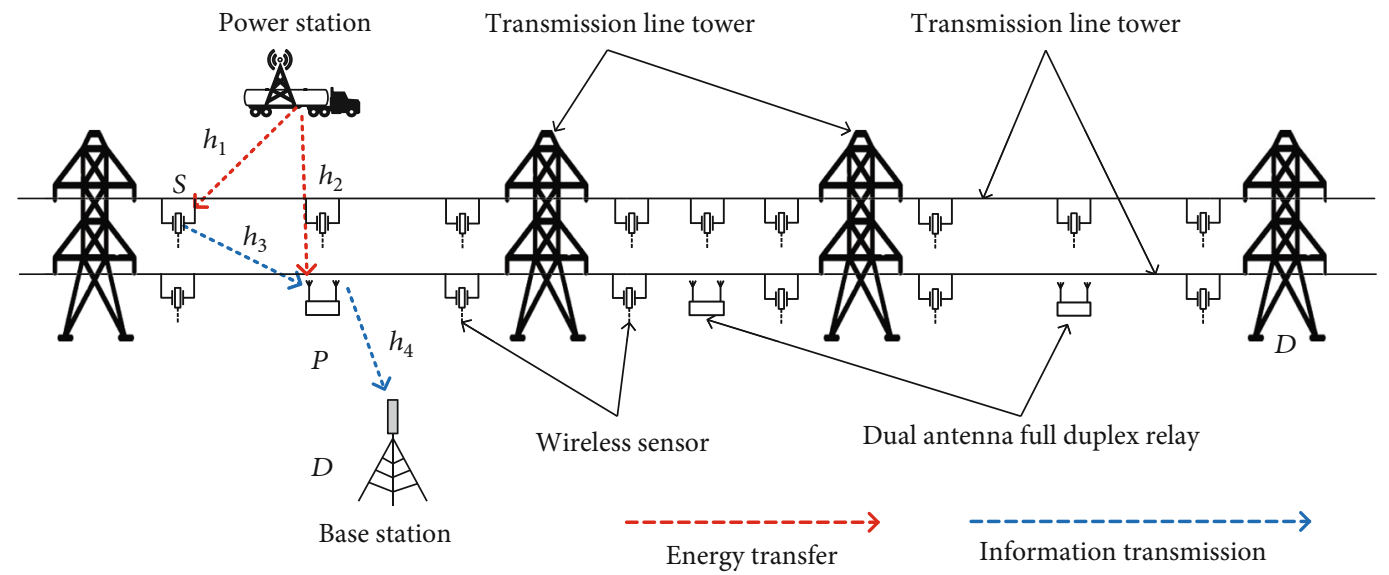

FIGURE 1: System model of full-duplex relay network in UEIoT.

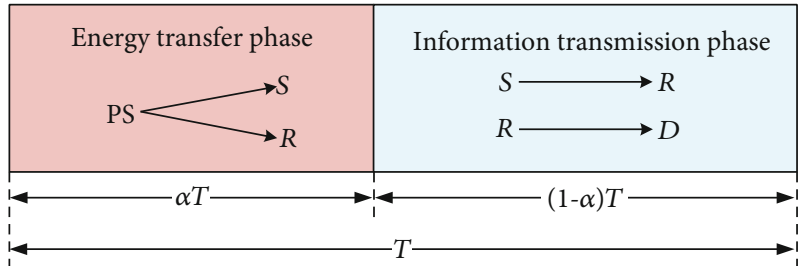

FIgURE 2: Time frame of the transmission protocol.

$$
E_{R}=\alpha T \eta P_{B} d_{2}^{-m}\left|h_{2}\right|^{2},
$$

where $h_{1}$ and $h_{2}$ are the channel coefficients of the links from PS to $S$ (PS-S) and from PS to $R$ (PS-R), respectively. $d_{1}$ and $d_{2}$ denote the distances between PS and $S$ and between PS and $R$, respectively. $P_{B}$ denotes the transmit power of PS. $m$ represents the path loss exponent. $\eta$ is the energy harvesting efficiency satisfying $0 \leq \eta \leq 1$.

During the second part with time interval of $(1-\alpha) T$, by using the harvested energies, $S$ and $R$ cooperatively transmit information to $D$ with the full-duplex mode; that is, $S$ transmits information to $R$, and $R$ simultaneously receives information of $S$ and forwards the information to $D$. From (1) and (2), the available transmit power at $S$ and $R$ can be, respectively, expressed by

$$
\begin{gathered}
P_{S}=\frac{E_{S}}{(1-\alpha) T}=\frac{\eta \alpha P_{B} d_{1}^{-m}\left|h_{1}\right|^{2}}{(1-\alpha)}, \\
P_{R}=\frac{E_{R}}{(1-\alpha) T}=\frac{\eta \alpha P_{B} d_{2}^{-m}\left|h_{2}\right|^{2}}{(1-\alpha)} .
\end{gathered}
$$

Let $x_{S}$ and $x_{R}$, respectively, represent the signals transmitted by $S$ and $R$, with $E\left[\left|x_{S}\right|^{2}\right]=P_{S}$ and $E\left[\left|x_{R}\right|^{2}\right]=P_{R} \cdot n_{R}$ denotes the additive Gaussian white noise (AWGN) with the mean of 0 and the variance of $N_{0}$ at the relay node $R$. The signal received at $R$ can be expressed as

$$
y_{R}=\sqrt{d_{3}^{-m}} h_{3} x_{S}+h_{R R} x_{R}+n_{R}
$$

where the first term of right part $\sqrt{d_{3}^{-m}} h_{3} x_{S}$ is the desired signal, and the second term $h_{R R} x_{R}$ is the self interference (SI). $h_{3}$ represents fading coefficient of the link from $S$ to $R(\mathrm{~S}-\mathrm{R}) . h_{R R}$ represents the channel coefficient of the link between the transmitting antenna and receiving antenna of $R . d_{3}$ is the distance between $S$ and $R$.

$R$ adopts self-interference cancellation (SIC) technology to eliminate SI. However, it is hard to completely eliminate SI in practice, and therefore, the residual self-interference (RSI) always exists [23], which may limit the system performance of full-duplex mode. In order to describe the RSI, denote it as $n_{R R}$, and then the signal received at $R$ can be transformed into [24]

$$
y_{R}=\sqrt{d_{3}^{-m}} h_{3} x_{S}+n_{R R}+n_{R}
$$

where $n_{R R}$ is modeled as a complex Gaussian distribution with mean of 0 and variance of $N_{R S I}$ [25]. $N_{R S I}$ is proportional to the transmit power $P_{R}$ of $R$, that is,

$$
N_{R S I}=\Omega P_{R}
$$

where $\Omega$ represents the SIC capability and indicates the strength of the self-interference cancellation capability of relay. The smaller the value $\Omega$ is, the stronger the relay SIC capability is.

Simultaneously, $R$ also decodes and forwards signal to $D$ while receiving the signal from $S$. The signal received at $D$ can be expressed as

$$
y_{D}=\sqrt{d_{4}^{-m}} h_{4} x_{R}+n_{D}
$$

where $h_{4}$ denotes the fading coefficient of the link from $R$ to $D(R-D)$, and $d_{4}$ represents the distance between $R$ and $D$. $n_{D}$ represents the AWGN with the mean of 0 and variance of $N_{0}$ at $D$. 
Following (6), the received signal-to-interference and noise ratio (SINR) for decoding at $R$ can be given by

$$
\gamma_{R}=\frac{d_{3}^{-m} P_{S}\left|h_{3}\right|^{2}}{N_{R S I}+N_{0}}
$$

By substituting (3) and (7) into (9), and using some simple mathematical transformations, we can obtain that

$$
\gamma_{R}=\frac{d_{1}^{-m} d_{3}^{-m} \varphi_{1} \varphi_{3}}{\Omega d_{2}^{-m} \varphi_{2}+(1 / \Phi)}
$$

where $\Phi=\eta \alpha \gamma_{0} /(1-\alpha)$ with $\gamma_{0}=P_{B} / N_{0}$, and $\varphi_{i}=\left|h_{i}\right|^{2}(i=1$ $, 2,3,4)$.

Similarly, by using (4), (7), and (8), and after some mathematical transformations, the received signal-to-noise ratio (SNR) at $D$ can be given by

$$
\gamma_{D}=\Phi d_{2}^{-m} d_{4}^{-m} \varphi_{2} \varphi_{4}
$$

\section{Outage Performance Analysis}

In this section, we analyze the outage probability of the system. As $R$ adopts DF protocol, it will decode the information of $S$ and forward the information to $D$ if the information is correctly decoded. Let $\gamma_{t h}$ be the required SINR for successful transmissions for both $S-R$ link and $R-D$ link. Then, the outage probability of the system can be expressed by

$$
P_{\text {out }}=P_{r}\left\{\gamma_{R}<\gamma_{t h}\right\}+\left(1-P_{r}\left\{\gamma_{R}<\gamma_{t h}\right\}\right) P_{r}\left\{\gamma_{D}<\gamma_{t h}\right\} \text {, }
$$

where $P_{r}\left\{\gamma_{R}<\gamma_{t h}\right\}$ is the outage probability of the first hop (i.e., $S-R$ link), and $P_{r}\left\{\gamma_{D}<\gamma_{t h}\right\}$ is the outage probability of the second hop (i.e., $R-D$ link). For convenience, $P_{r}\left\{\gamma_{R}<\gamma_{t h}\right\}$ and $P_{r}\left\{\gamma_{D}<\gamma_{t h}\right\}$ are represented as $P_{\text {out } \_R}$ and $P_{\text {out } \_D}$, respectively.

Define $R_{\text {th }}$ (in bps/Hz) as a target transmission rate threshold. According to the Shannon formula, we can have that

$$
R_{t h}=(1-\alpha) \log _{2}\left(1+\gamma_{t h}\right)
$$

and thus $\gamma_{t h}$ can be calculated as $\gamma_{t h}=2^{R_{t h} /(1-\alpha)}-1$.

To calculate $P_{\text {out_R }}$ and $P_{\text {out_ } D}$, firstly it can be seen that there are two multiplied random variables $\varphi_{i}$ in (10) and (11). Following the Rician distribution, the probability density function (PDF) $f_{\varphi_{i}}(x)$ and cumulative probability function $(\mathrm{CDF}) F_{\varphi_{i}}(x)$ of the random variable $\varphi_{i}$ are, respectively, given by [26]

$$
f_{\varphi_{i}}(x)=\frac{\left(K_{i}+1\right) e^{-K_{i}}}{\lambda_{i}} \sum_{l=0}^{\infty}\left(\frac{K_{i}+1}{\lambda_{i}}\right)^{l} \frac{K_{i}^{l} x^{l}}{(l !)^{2}} e^{-\frac{K_{i}+1}{\lambda_{i}} \cdot x},
$$

$$
F_{\varphi_{i}}(x)=\int_{0}^{x} f_{\varphi_{i}}(x) d x=1-e^{-K_{i}} \sum_{l=0}^{\infty} \sum_{m=0}^{l} \frac{K_{i}^{l} B_{i}{ }^{m} x^{m}}{m ! l !} e^{-B_{i} x}
$$

where $B_{i}=\left(\left(K_{i}+1\right) / \lambda_{i}\right)(i=1,2,3,4) . K_{i}$ and $\lambda_{i}$ represent the Rician factor and the mean of random variables $\varphi_{i}$, respectively.

In order to facilitate the derivation and analysis, define an auxiliary variable $Y_{i j}=\varphi_{i} \varphi_{j}$, and the CDF of $Y_{i j}$ can be expressed as

$$
\begin{aligned}
F_{Y_{\mathrm{ij}}}(y) & =P_{r}\left\{Y_{\mathrm{ij}}<y\right\}=P_{r}\left\{\varphi_{\mathrm{i}} \varphi_{\mathrm{j}}<y\right\} \\
& =P_{r}\left\{\varphi_{\mathrm{i}}<\frac{y}{\varphi_{\mathrm{j}}}\right\}=\int_{0}^{\infty} F_{\varphi_{\mathrm{i}}}\left(\frac{y}{\varphi_{\mathrm{j}}}\right) f_{\varphi_{\mathrm{j}}}\left(\varphi_{\mathrm{j}}\right) d \varphi_{\mathrm{j}} .
\end{aligned}
$$

By using (14) and (15), the CDF of $Y_{i j}$ in (16) can be further calculated as

$$
\begin{aligned}
F_{Y_{i j}}(y) & =\int_{0}^{\infty}\left\{1-e^{-K_{i}} \sum_{l=0}^{\infty} \sum_{m=0}^{l} \frac{K_{i}^{l} B_{i}^{m}}{l ! m !} \cdot\left(\frac{y}{\varphi_{j}}\right)^{m} \cdot e^{-B_{i} \cdot \frac{y}{\varphi_{j}}}\right\} f_{\varphi_{j}}\left(\varphi_{j}\right) d \varphi_{j} \\
& =1-\int_{0}^{\infty} e^{-K_{i}} \sum_{l=0}^{\infty} \sum_{q=0}^{l} \frac{K_{i}^{l} B_{i}^{q}}{l ! q !}\left(\frac{y}{\varphi_{j}}\right)^{q} e^{-B_{i} \cdot \frac{y}{\varphi_{j}}} A_{j} e^{-B_{j} \varphi_{j}} \sum_{k=0}^{\infty} \frac{\left(B_{j} K_{j}\right)^{k}}{(k !)^{2}} \cdot \varphi_{j}{ }^{k} d \varphi_{j} \\
= & 1-2 e^{-\left(K_{i}+K_{j}\right)} \sum_{l=0}^{\infty} \sum_{k=0}^{\infty} \sum_{q=0}^{l} \frac{K_{i}^{l} K_{j}^{k}}{l ! q !(k !)^{2}}\left(B_{i} B_{j} y\right)^{\frac{k+q+1}{2}} K_{k-q+1}\left(2 \sqrt{B_{i} B_{j} y}\right),
\end{aligned}
$$

where $A_{j}=B_{j} e^{-K_{i}}$ and $K_{v}(\cdot)$ are the modified Bessel function of the second kind [27].

Then, by using (10) and (17), the outage probability of the first hop can be derived as

$$
\begin{aligned}
P_{\text {out } \_R} & =P_{r}\left\{\frac{d_{1}^{-m} d_{3}^{-m} \varphi_{1} \varphi_{3}}{\Omega_{B} d_{2}^{-m} \varphi_{2}+(1 / \Phi)}<\gamma_{t h}\right\} \\
& =P_{r}\left\{Y_{13}<\frac{\gamma_{t h}\left(\Omega_{B} d_{2}^{-m} \varphi_{2}+(1 / \Phi)\right)}{d_{1}^{-m} d_{3}^{-m}}\right\} \\
& =\int_{0}^{\infty} f_{\varphi_{2}}\left(\varphi_{2}\right) F_{Y_{13}}\left(\frac{\gamma_{t h}\left(\Omega_{B} d_{2}^{-m} \varphi_{2}+(1 / \Phi)\right)}{d_{1}^{-m} d_{3}^{-m}}\right) d \varphi_{2} \\
& \triangleq 1-\Delta,
\end{aligned}
$$

where

$$
\begin{aligned}
\Delta= & 2 A_{2} e^{-\left(K_{1}+K_{3}\right)} \sum_{q=0}^{\infty} \sum_{l=0}^{\infty} \sum_{k=0}^{\infty} \sum_{z=0}^{l} \frac{\left(B_{2} K_{2}\right)^{q} K_{1}^{l} K_{3}{ }^{k}}{l ! z !(k !)^{2}(q !)^{2}} \\
& \cdot \int_{0}^{\infty} \varphi_{2}{ }^{q} e^{-B_{2} \varphi_{2}\left[\frac{B_{1} B_{3} \gamma_{t h}\left(\Omega_{B} d_{2}^{-m} \varphi_{2}+(1 / \Phi)\right)}{d_{1}^{-m} d_{3}^{-m}}\right]^{\frac{k+m+1}{2}}} \\
& \cdot K_{k-z+1}\left(2 \sqrt{\frac{B_{1} B_{3} \gamma_{t h}\left(\Omega d_{2}^{-m} \varphi_{2}+(1 / \Phi)\right)}{d_{1}^{-m} d_{3}^{-m}}}\right) d \varphi_{2} .
\end{aligned}
$$

Similarly, by using (11) and (17), the outage probability of the second hop can be derived as 
TABLE 1: System simulation parameter table.

\begin{tabular}{lcc}
\hline Parameter & Explanation & Default value/value range \\
\hline$\eta$ & Energy harvesting efficiency & $0.8 / 0-1$ \\
$\lambda_{i}$ & Mean of $\varphi_{i}$ & 1 \\
$d_{i}$ & Distance & $3 \mathrm{~m}$ \\
$m$ & Path loss coefficient & 2 \\
$\Omega$ & SIC capability & $-20 \mathrm{~dB}$ \\
$R_{\mathrm{th}}$ & Transmission rate & $0.05 \mathrm{bits} / \mathrm{s} / \mathrm{Hz} / 0.01-0.5 \mathrm{bits} / \mathrm{s} / \mathrm{Hz}$ \\
$\alpha$ & Time division factor & $0.4 / 0-1$ \\
$\gamma_{0}$ & Power station transmit power and noise power ratio $P_{B} / N_{0}$ & $0-50 \mathrm{~dB}$ \\
$K_{i}$ & Rician factor & $2 / 0-4$ \\
\hline
\end{tabular}

$$
P_{\text {out } \_D}=F_{Y_{24}}\left(\frac{\gamma_{t h}}{\Phi d_{2}^{-m} d_{4}^{-m}}\right) \triangleq 1-\Theta
$$

where

$$
\Theta=2 e^{-\left(K_{2}+K_{4}\right)} \sum_{p=0}^{\infty} \sum_{t=0}^{\infty} \sum_{n=0}^{p} \frac{K_{2}^{p} K_{4}{ }^{t}}{p ! n !(t !)^{2}}\left(\frac{B_{2} B_{4} \gamma_{t h}}{\Phi d_{2}^{-m} d_{4}^{-m}}\right)^{\frac{t+n+1}{2}} K_{t-n+1}\left(2 \sqrt{\frac{B_{2} B_{4} \gamma_{t h}}{\Phi d_{2}^{-m} d_{4}^{-m}}}\right) .
$$

Substituting (18) and (20) into (12), we can obtain the outage probability of system as

$$
\begin{aligned}
P_{\text {out }} & =P_{r}\left\{\gamma_{R}<\gamma_{t h}\right\}+\left(1-P_{r}\left\{\gamma_{R}<\gamma_{t h}\right\}\right) P_{r}\left\{\gamma_{D}<\gamma_{t h}\right\} \\
& =(1-\Delta)+(1-(1-\Delta))(1-\Theta)=1-\Delta \Theta
\end{aligned}
$$

So, by substituting (19) and (21) into (22), the outage probability of the system is expressed as

$$
\begin{aligned}
& P_{\text {out }}=1-4 A_{2} e^{-\left(K_{1}+K_{2}+K_{3}+K_{4}\right)} \sum_{q=0}^{\infty} \sum_{l=0}^{\infty} \sum_{k=0}^{\infty} \sum_{z=0}^{l} \frac{\left(B_{2} K_{2}\right)^{q} K_{1}^{l} K_{3}{ }^{k}}{l ! z !(k !)^{2}(q !)^{2}} \\
& \cdot \int_{0}^{\infty} \varphi_{2}{ }^{q} e^{-B_{2} \varphi_{2}}\left[\frac{B_{1} B_{3} \gamma_{t h}\left(\Omega d_{2}^{-m} \varphi_{2}+(1 / \Phi)\right)}{d_{1}^{-m} d_{3}^{-m}}\right]^{\frac{k+z+1}{2}} \\
& \cdot K_{k-z+1}\left(2 \sqrt{\frac{B_{1} B_{3} \gamma_{t h}\left(\Omega d_{2}^{-m} \varphi_{2}+(1 / \Phi)\right)}{d_{1}^{-m} d_{3}^{-m}}}\right) d \varphi_{2} \\
& \cdot \sum_{p=0}^{\infty} \sum_{t=0}^{\infty} \sum_{n=0}^{p} \frac{K_{2}^{p} K_{4}{ }^{t}}{p ! n !(t !)^{2}}\left[\frac{B_{2} B_{4} \gamma_{t h}}{\Phi d_{2}^{-m} d_{4}^{-m}}\right]^{\frac{t+n+1}{2}} \\
& K_{t-n+1}\left(2 \sqrt{\frac{B_{2} B_{4} \gamma_{t h}}{\Phi d_{2}^{-m} d_{4}^{-m}}}\right)
\end{aligned}
$$

Finally, the system throughput for a delay limited scenario can be expressed as

$$
R=R_{\mathrm{th}}\left(1-P_{\text {out }}\right)(1-\alpha)
$$

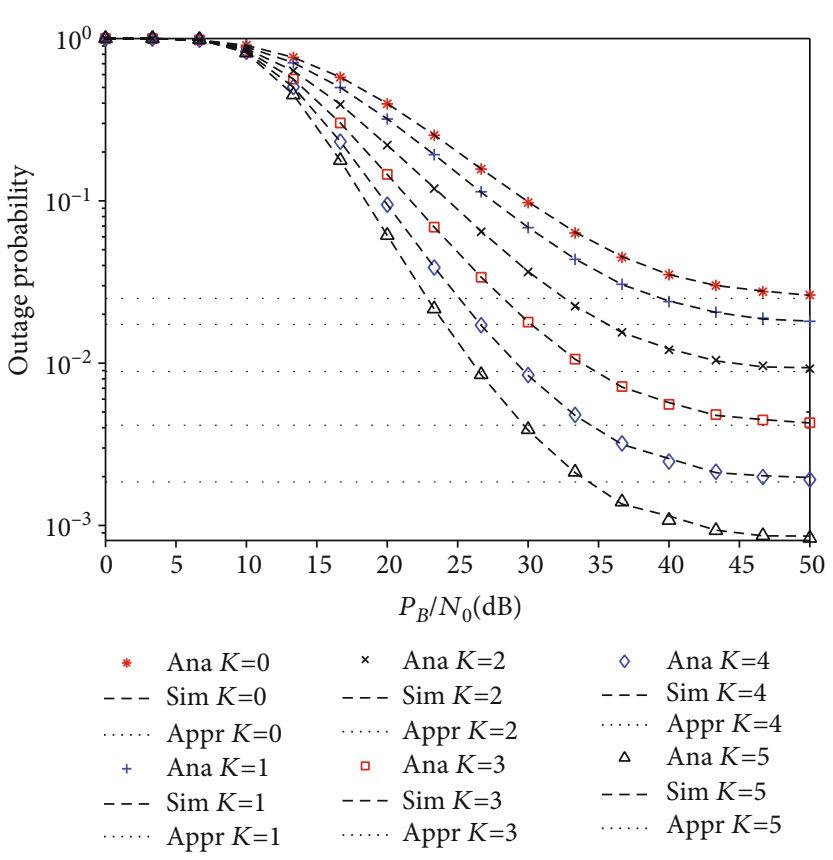

FIgURe 3: The system outage probability versus $P_{B} / N_{0}$ for different Rician factor $K$.

\section{Simulation Result}

This section presents some simulation results to verify our obtained theoretical results and demonstrate the system performances. For clarity, the simulation parameters are listed in Table 1. In the figures of the section, the marker of Ana \pm denotes the numerical results calculated by the derived closed expression, the marker of $\mathrm{Sim} \pm$ denotes the simulation results obtained by Monte Carlo method, and Appr \pm denotes the numerical asymptote in high SNR regime. In the following, we will discuss the effect of system parameters and PS position on the system performance, respectively.

4.1. The Effect of System Parameters on Performance. Figures 3 and 4 show the effect of the ratio of the PS transmit power to the noise power (i.e., $\gamma_{0}=P_{B} / N_{0}$ ) on the system outage probability and throughput with different values of the 


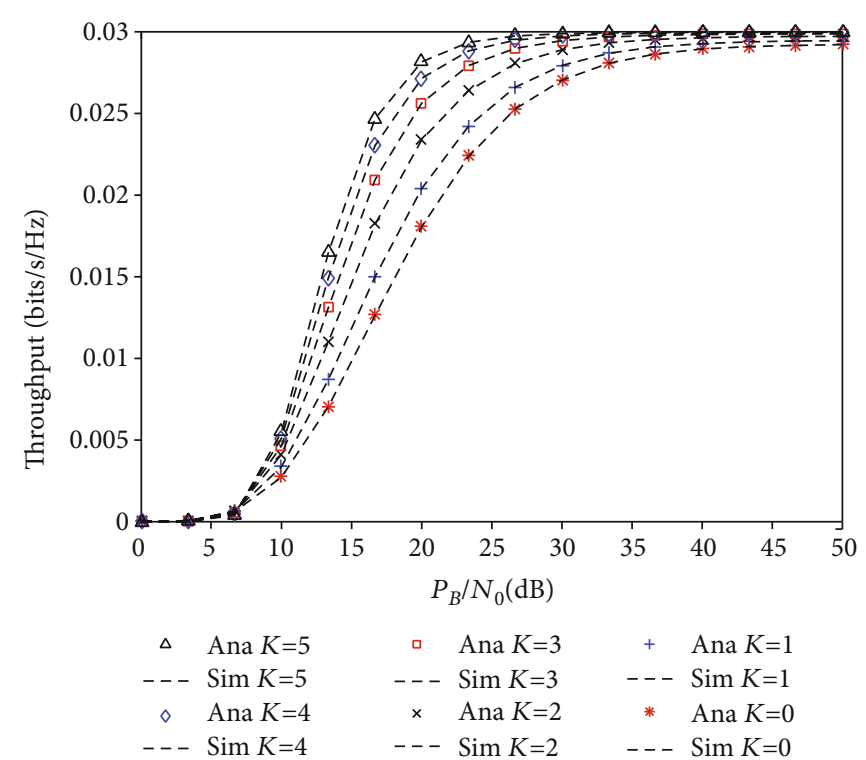

Figure 4: The system throughput versus $P_{B} / N_{0}$ for different Rician factor $K$.

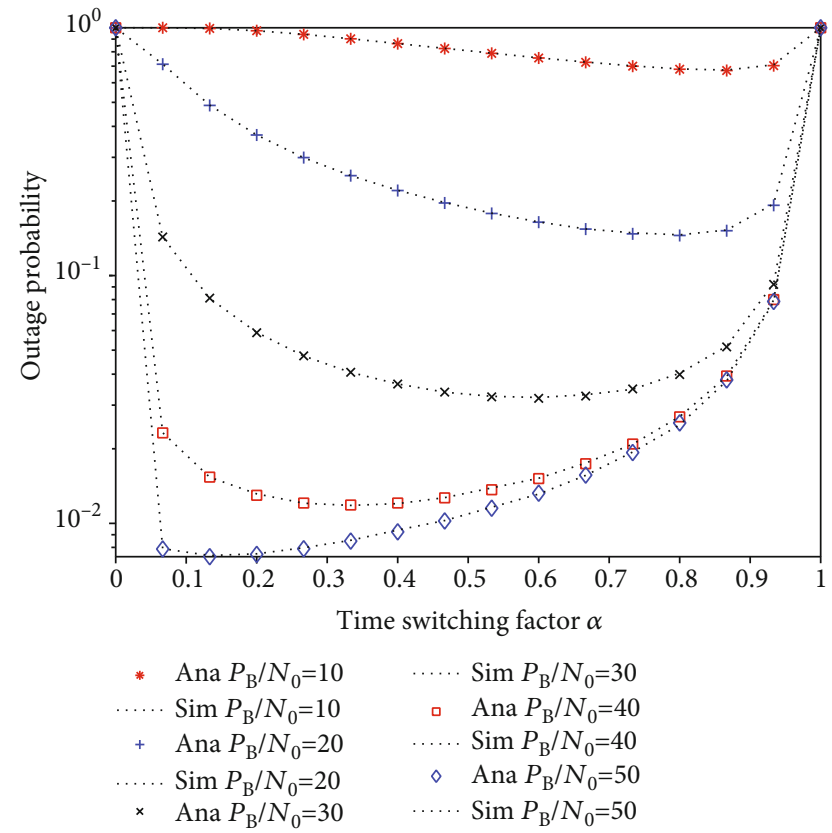

Figure 5: The system outage probability versus time switching factor $\alpha$ for different $\gamma_{0}$.

Rician factor, respectively. From Figures 3 and 4, it can be seen that the results calculated by the closed expression match the results obtained by Monte Carlo simulation well, which demonstrates the correctness of our obtained theoretical results.

From Figures 3 and 4, one can also find that for a fixed Rician factor $K$, the greater the value of $\gamma_{0}$ is, the smaller the system outage probability becomes, and the larger the system throughput becomes. This is because with the increment of

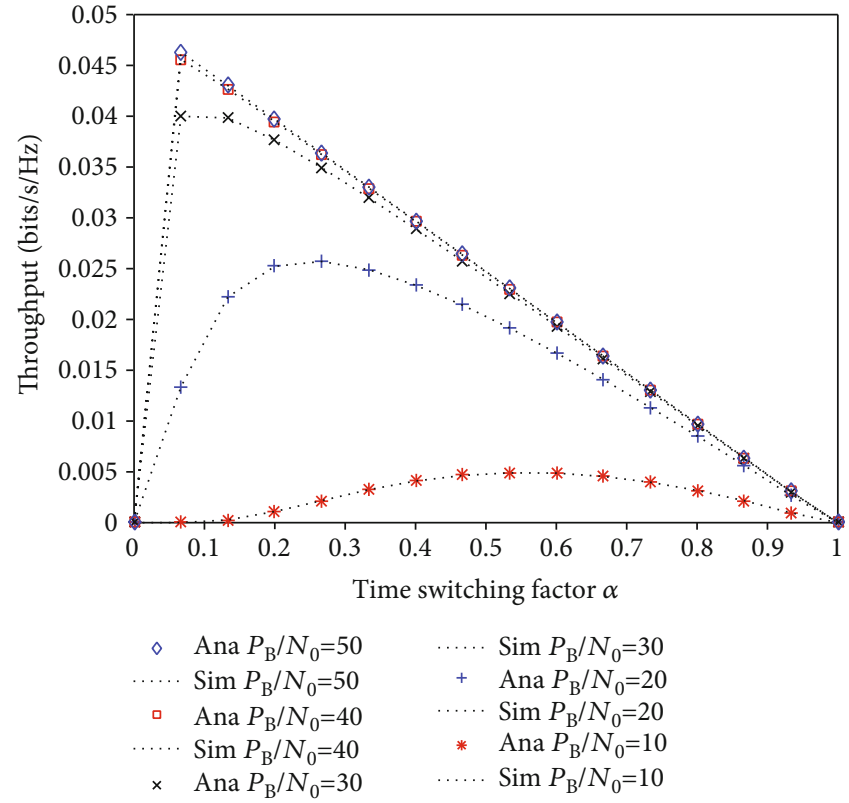

FIgURE 6: The system throughput versus time switching factor $\alpha$ for different $\gamma_{0}$.

$\gamma_{0}$ for given $N_{0}$, the energy harvested by $S$ and $R$ increases, which yields higher SINR of both $R$ and $D$. It can also be found that for fixed $\gamma_{0}$, the greater the value of the Rician factor is, the lower the outage probability of the system becomes, and the larger the system throughput becomes. The reason is that the larger Rician factor means the stronger LOS, which increases the energy harvested by both $S$ and $R$. Finally, from Figure 3, it can be seen that the outage probability gradually approaches an numerical asymptote in high SNR regime. This indicates that increasing the transmit power of PS cannot infinitely decrease the outage probability of the system.

Figures 5 and 6 show the effects of the time division factor $\alpha$ on the system outage probability and throughput for different values of $\gamma_{0}$, respectively. The results calculated by the closed expression fit well with those obtained by Monte Carlo simulation, confirming that the derived closed-form solution of the system outage probability is completely accurate again. One can see that for a fixed $\alpha$, the larger $\gamma_{0}$ is, the lower outage probability is and the larger throughput is, which agrees with Figures 3 and 4.

From Figure 5, one can see that for a fixed $\gamma_{0}$, with the increment of the value of $\alpha$, the outage probability decreases first and then increases. On one hand, when the value of $\alpha$ is close to 0 , the time allocated for energy harvesting is relatively short. In this case, the less energy can be harvested by $S$ and $R$ and therefore, the outage probability of system is close to 1 . On the other hand, when the value of $\alpha$ approaches to 1 , the time assigned for information relaying transmission becomes very short, and thus the outage probability of system tends to be 1 . In addition, it can be found that there is an optimal value of $\alpha$, which makes the outage probability of system reach the lowest. For example, for $\gamma_{0}=30$, when $\alpha$ is equal to about 0.6 , the system achieves the best outage performance. 


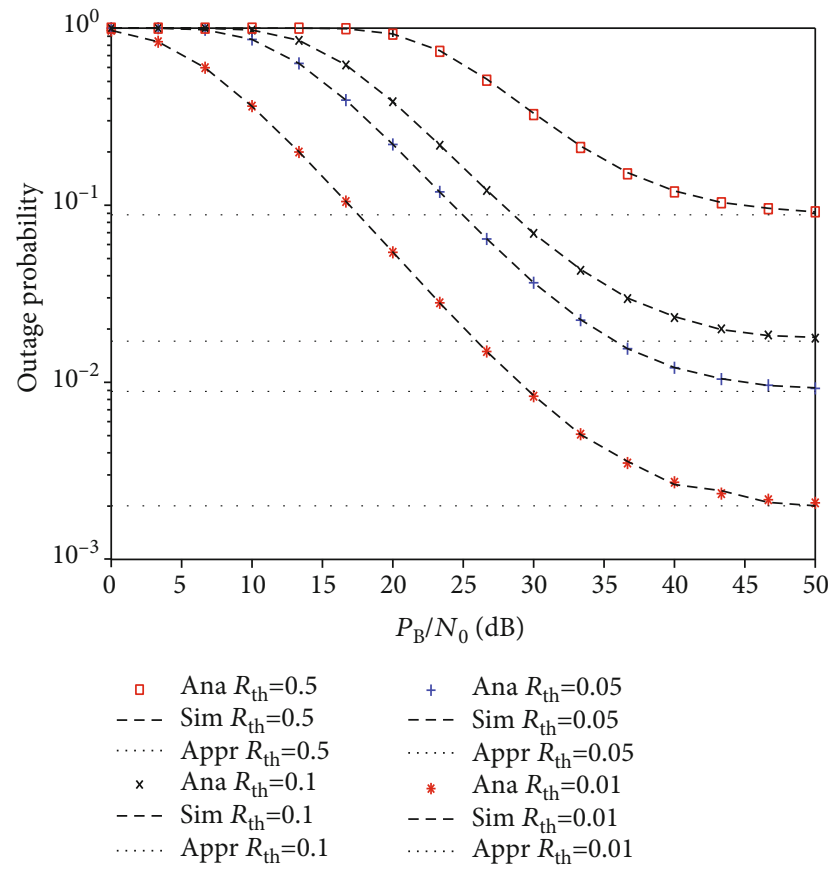

Figure 7: The system outage probability versus $P_{B} / N_{0}$ for different transmission rate threshold $R_{t h}$.

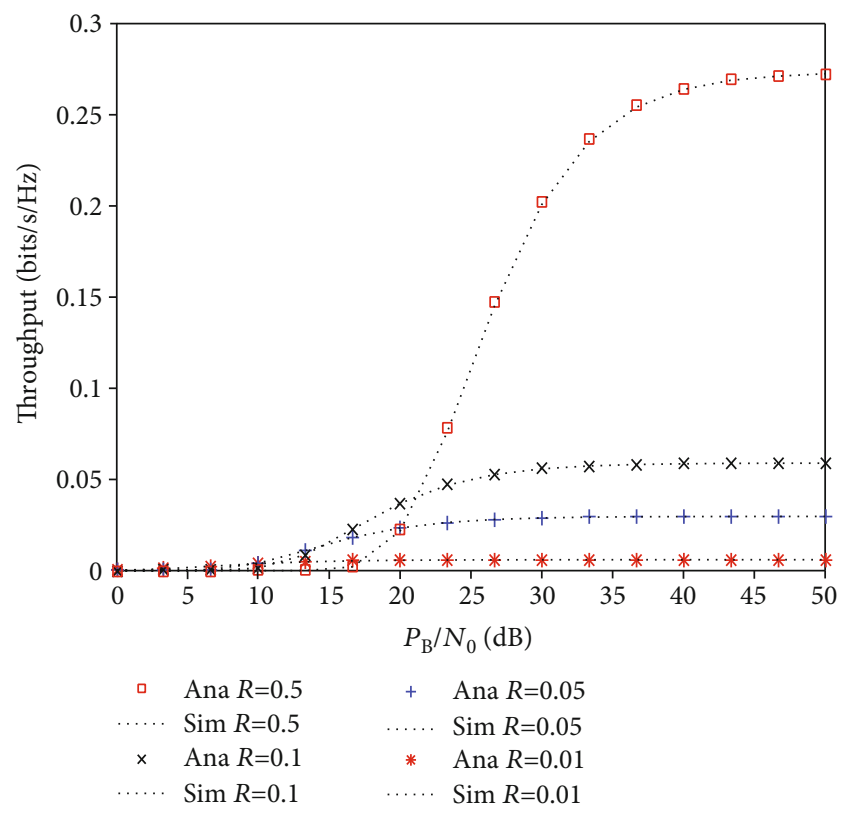

Figure 8: The system throughput versus $P_{B} / N_{0}$ for different transmission rate threshold $R_{t h}$.

From Figure 6 , one can also see that for a fixed $\gamma_{0}$, with the increment of the value of $\alpha$, the system throughput increases first and then decreases. The reason is as follows. When the value of $\alpha$ is close to $0, S$ and $R$ do not have energy to transmit information, while when the value of $\alpha$ is close to $1, S$ and $R$ do not have time to transmit information. Moreover, it can be found that there is an optimal value of $\alpha$, which makes the system throughput reach the largest. For example, for $\gamma_{0}=30$,

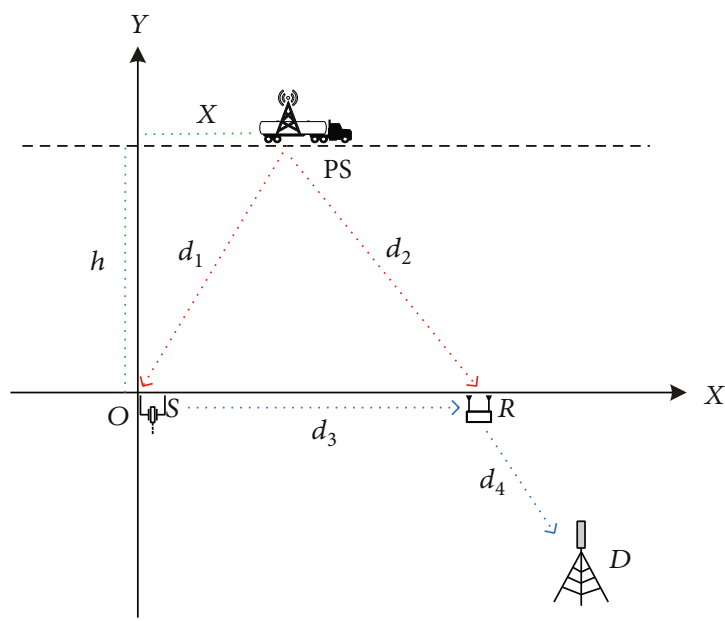

FIgURE 9: The considered coordinate system.

when $\alpha$ is equal to about 0.08 , the system achieves the best throughput performance. Finally, by comparing Figure 6 with Figure 5, it can be found that the optimal values of $\alpha$ are different for the best outage probability and throughput.

Figures 7 and 8 discuss the effect of transmission rate threshold $R_{t h}$ on the system outage probability and throughput with respect to $\gamma_{0}$, respectively. It can be seen that for fixed transmission rate threshold $R_{t h}$, as the value of $\gamma_{0}$ increases, the outage probability gradually decreases, and the throughput gradually increases, and they tend to be stable, which is consistent with the results in Figures 3 and 4 . Moreover, from Figure 7, one can observe that for a fixed $\gamma_{0}$, the smaller the value of $R_{t h}$ is, the lower the value of system outage probability becomes. This is because when $R_{t h}$ becomes smaller, $\gamma_{t h}=2^{R /(1-\alpha)}-1$ also becomes smaller, which further causes lower outage probability. Finally, from Figure 8, it can found that the larger the value of $R_{t h}$ is, the larger the system throughput becomes. The reason is that from (24) one can see that with the decrement of system outage probability, the throughout gradually increases.

4.2. The Effect of Power Station Position on Performance. In order to discuss the effect of power station position on performance, we consider a coordinate system, as shown in Figure 9. $S$ is located at the origin of coordinate, $R$ is located at the positive $X$-axis, and their distance $d_{3}$ is set to be $10 \mathrm{~m}$. $D$ is located in the fourth quadrant and its distance $d_{4}$ from $R$ may be set to $5 \mathrm{~m}, 10 \mathrm{~m}, 20 \mathrm{~m}$, or $30 \mathrm{~m}$. PS moves at a straight line whose distance $h$ from the $X$-axis is set to be $10 \mathrm{~m}$. The $X$ -axis coordinate of PS is denoted by $x$, which is used to represent the position of PS. Besides, the ratio of PS transmit power to the noise power $\gamma_{0}$ is set to be $40 \mathrm{~dB}$, and other system parameters are set according to Table 1 .

Figures 10 and 11 show the system outage probability and system throughput with respect to PS position $x$ for different distances between $R$ and $D d_{4}$, respectively. It can be found that with the increment of the $X$-axis coordinate of PS $x$, the outage probability first decreases and then increases while the throughput first increases and then decreases. And there 


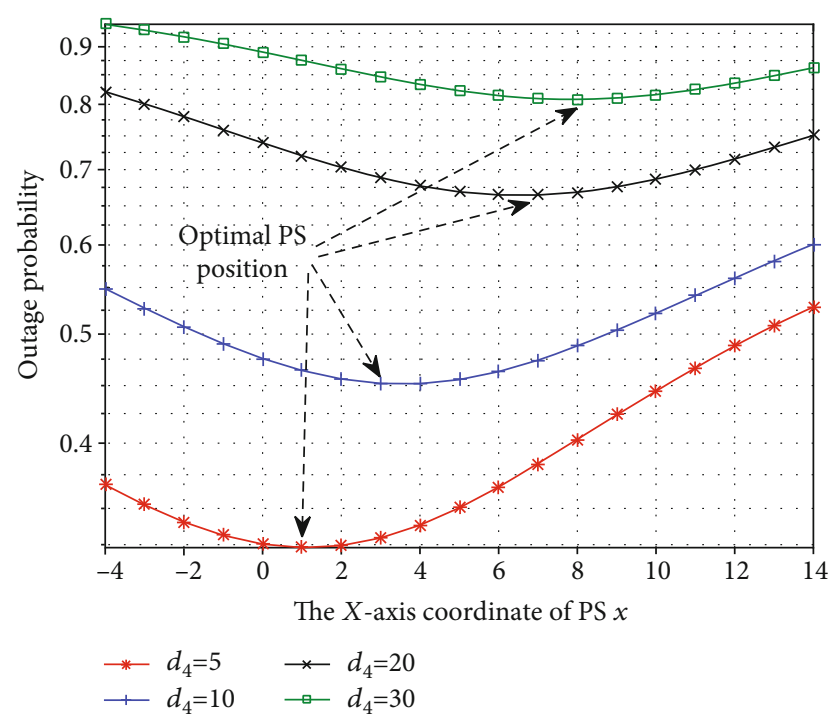

Figure 10: The system outage probability versus PS position $x$ for different distances between $R$ and $D d_{4}$.

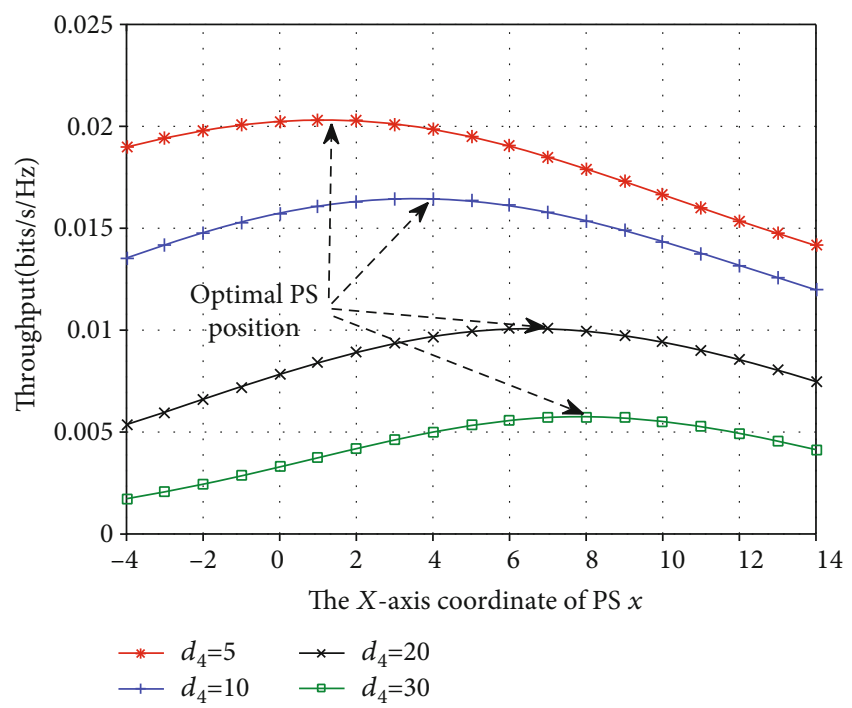

FIGURE 11: The system throughput versus PS position $x$ for different distances between $R$ and $D d_{4}$.

is an optimal PS position whose $X$-axis coordinate is located between $S$ and $R$, which makes the outage probability of system reaches the lowest or system throughput reach the largest. The reason is when the $X$-axis coordinate of PS is located between $S$ and $R$, and both hops are balanced, which leads to lower outage probability and larger throughput. It can be also seen that with the increment of distance between $R$ and $D d_{4}$, the outage probability increases, and the throughput decreases, which is caused by larger path loss from $R$ to $D$ . Finally, one can see that with the increment of distance between $R$ and $D d_{4}$, the optimal PS position is gradually close to $R$. This is because when PS is closer to $R$, it can supply more energy to $R$ to alleviate the effect of larger path loss from $R$ to $D$.

\section{Conclusion}

This paper has studied the communication outage performance of full-duplex relay networks powered by RF energy harvesting in UEIoT, where the outdoor Rician fading channel model is adopted and full-duplex relaying protocol is employed. The explicit expression of the outage probability of the system has been derived. Simulation results have validated the correctness of our theoretical analysis and also evaluated the effects of the transmit power of the power station, the Rician factor, the time division factor, and system transmission rate threshold on the system outage probability and throughput. The optimal deployment position of power station is also investigated, which provides some useful insights on the future practical applications of UEIoT.

\section{Data Availability}

The data used to support the findings of this study are available from the corresponding author upon request.

\section{Conflicts of Interest}

The authors declare that they have no conflicts of interest.

\section{Acknowledgments}

This work was supported by the National Natural Science Foundation of China under Grant (no. 61801155) and was supported by the Self-Developed Project of State Grid Energy Research Institute Co., Ltd (Ubiquitous Electric Internet of Things Edge Computing Performance Analysis and Simulation Based on Typical Scenarios, SGNY202009014).

\section{References}

[1] Z. Zhai, L. Jia, Y. Wang, Y. Ma, W. Jing, and Z. Zhang, "Research on ubiquitous Power Internet of Things architecture," in 2019 IEEE 3rd Conference on Energy Internet and Energy System Integration (EI2), pp. 435-439, Changsha, China, 2019.

[2] The State Grid Corporation of China, "White paper: Internet of Things in electricity," 2019, http://news.bjx.com.cn/html/ 20191014/1012993.shtml.

[3] The State Grid Corporation of China, "The state grid outline for the construction of ubiquitous power Internet of Things was officially released," 2019, http://www.chinasmartgrid .com.cn/news/20190311/632172.shtml.

[4] Y. Gong, C. Chen, B. Liu, G. Gong, B. Zhou, and N. K. Mahato, "Research on the ubiquitous electric power internet of things security management based on edge-cloud computing collaboration technology," in 2019 IEEE Sustainable Power and Energy Conference (iSPEC), pp. 1997-2002, Beijing, China, 2019.

[5] Q. Wang and Y. G. Wang, "Research on Power Internet of Things architecture for smart grid demand," in 2018 2nd IEEE Conference on Energy Internet and Energy System Integration (EI2), pp. 1-9, Beijing, China, 2018.

[6] K. Xiong, C. Chen, G. Qu, P. Fan, and K. B. Letaief, "Group cooperation with optimal resource allocation in wireless 
powered communication networks," IEEE Transactions on Wireless Communications, vol. 16, no. 6, pp. 3840-3853, 2017.

[7] K. W. Choi, L. Ginting, P. A. Rosyady, A. A. Aziz, and D. I. Kim, "Wireless-powered sensor networks: how to realize," IEEE Transactions on Wireless Communications, vol. 16, no. 1, pp. 221-234, 2017.

[8] J. Liu, K. Xiong, P. Fan, and Z. Zhong, "RF energy harvesting wireless powered sensor networks for smart cities," IEEE Access, vol. 5, pp. 9348-9358, 2017.

[9] X. Li, G. Zhu, Y. Gong, and K. Huang, "Wirelessly powered data aggregation for IoT via over-the-air function computation: beamforming and power control," IEEE Transactions on Wireless Communications, vol. 18, no. 7, pp. 3437-3452, 2019.

[10] D. S. Gurjar, H. H. Nguyen, and P. Pattanayak, "Performance of wireless powered cognitive radio sensor networks with nonlinear energy harvester," IEEE Sensors Letters, vol. 3, no. 8, pp. 1-4, 2019.

[11] Y. Liu, Y. Ye, H. Ding, F. Gao, and H. Yang, "Outage performance analysis for SWIPT-based incremental cooperative NOMA networks with non-linear harvester," IEEE Communications Letters, vol. 24, no. 2, pp. 287-291, 2020.

[12] D. Lecce, A. Grassi, G. Piro, and G. Boggia, "Boosting energy efficiency of NB-IoT cellular networks through cooperative relaying," in 2018 IEEE 29th Annual International Symposium on Personal, Indoor and Mobile Radio Communications (PIMRC), pp. 1-5, Bologna, Italy, 2018.

[13] T. Riihonen, S. Werner, and R. Wichman, "Comparison of full-duplex and half-duplex modes with a fixed amplify-andforward relay," in 2009 IEEE Wireless Communications and Networking Conference, pp. 1-5, Budapest, Hungary, 2009.

[14] R. Nikjah and N. Beaulieu, "Achievable rates and fairness in rateless coded decode-and-forward half-duplex and fullduplex opportunistic relaying," in 2008 IEEE International Conference on Communications, pp. 3701-3707, Beijing, China, 2008.

[15] K. Kwon, D. Hwang, and S. S. Nam, "Beamformer design for self-energy recycling in full-duplex decode-and-forward relay systems," IEEE Wireless Communications Letters, vol. 9, no. 9, pp. 1417-1421, 2020.

[16] J.-H. Lee, S. S. Nam, and Y.-C. Ko, "Outage performance analysis of two-way full-duplex DF relaying networks with beamforming," IEEE Transactions on Vehicular Technology, vol. 69, no. 8, pp. 8753-8763, 2020.

[17] L. Samara, A. Gouissem, R. Hamila, M. O. Hasna, and N. AlDhahir, "Full-duplex amplify-and-forward relaying under I/Q imbalance," IEEE Transactions on Vehicular Technology, vol. 69, no. 7, pp. 7966-7970, 2020.

[18] X. Song and S. Xu, "Joint optimal power allocation and relay selection in full-duplex energy harvesting relay networks," in 2018 10th International Conference on Communication Software and Networks (ICCSN), pp. 80-84, Chengdu, China, 2018.

[19] G. Chen, P. Xiao, J. R. Kelly, B. Li, and R. Tafazolli, "Fullduplex wireless-powered relay in two way cooperative networks," IEEE Access, vol. 5, pp. 1548-1558, 2017.

[20] Z. Wei, S. Sun, X. Zhu, D. in Kim, and D. W. K. Ng, "Resource allocation for wireless-powered full-duplex relaying systems with nonlinear energy harvesting efficiency," IEEE Transactions on Vehicular Technology, vol. 68, no. 12, pp. 1207912093, 2019.
[21] B. C. Nguyen, T. M. Hoang, and P. T. Tran, "Performance analysis of full-duplex decode-and-forward relay system with energy harvesting over Nakagami- _ $\mathrm{m}_{-}$fading channels," AEU - International Journal of Electronics and Communications, vol. 98, pp. 114-122, 2019.

[22] S. Kumar, S. De, and D. Mishra, "RF energy transfer channel models for sustainable IoT," IEEE Internet of Things Journal, vol. 5, no. 4, pp. 2817-2828, 2018.

[23] K. Yang, H. Cui, L. Song, and Y. Li, "Efficient full-duplex relaying with joint antenna-relay selection and self-interference suppression," IEEE Transactions on Wireless Communications, vol. 14, no. 7, pp. 3991-4005, 2015.

[24] Q. Wang, Y. Dong, X. Xu, and X. Tao, “Outage probability of full-duplex AF relaying with processing delay and residual self-interference," IEEE Communications Letters, vol. 19, no. 5, pp. 783-786, 2015.

[25] M. Duarte, C. Dick, and A. Sabharwal, "Experiment-driven characterization of full-duplex wireless systems," IEEE Transactions on Wireless Communications, vol. 11, no. 12, pp. 4296-4307, 2012.

[26] G. L. Stueber, Principles of Mobile Communication, Springer, Norwell, MA USA, 2001.

[27] I. S. Gradshteyn and I. M. Ryzhik, "Table of integrals series and products," Academic press, New York, NY, USA, 2007. 